\title{
Choroidal vessel density in unilateral hyperopic amblyopia using en-face optical coherence tomography
}

Syunsuke Araki ${ }^{1,2}$, Atsushi Miki 1,3,4, ${ }^{*}$, Katsutoshi Goto ${ }^{1}$, Tsutomu Yamashita ${ }^{1,3}$, Tsuyoshi Yoneda ${ }^{1,3}$, Atsushi Fujiwara ${ }^{1,3}$, Kazuko Haruishi ${ }^{1,3}$, Yoshiaki leki ${ }^{1}$, Junichi Kiryu ${ }^{1}$, Goro Maehara ${ }^{6}$ and Kiyoshi Yaoeda ${ }^{4,5,7}$

\begin{abstract}
Background: Structural changes of the choroid, such as choroidal thickening, have been indicated in amblyopic eyes with hyperopic anisometropia as compared to fellow or healthy eyes. The purpose of the present study was to investigate choroidal vascular density (CVD) in children with unilateral hyperopic amblyopia.

Methods: This study included 88 eyes of 44 patients with unilateral amblyopia due to hyperopic anisometropia with or without strabismus and 29 eyes of 29 age-matched normal controls. The CVD of Haller's layer was quantified from en-face images constructed by 3-dimensional swept-source optical coherence tomography images flattened relative to Bruch's membrane. The analysis area was a $3 \times 3-\mathrm{mm}$ square of macula after magnification correction. Relationships between CVD and other parameters [best-corrected visual acuity (BCVA), refractive error and subfoveal choroidal thickness (SFCT)] were investigated, and CVDs were compared between amblyopic, fellow, and normal control eyes.

Results: Mean CVD was $59.11 \pm 0.66 \%$ in amblyopic eyes, $59.23 \pm 0.81 \%$ in fellow eyes, and $59.29 \pm 0.74 \%$ in normal control eyes. CVD showed a significant positive relationship with SFCT $(p=0.004)$, but no relationships with other parameters. No significant differences in CVD were evident among amblyopic, fellow, and normal control eyes after adjusting for SFCT $(p=0.502)$.

Conclusions: CVD was unrelated to BCVA, and CVD did not differ significantly among amblyopic, fellow and normal control eyes. These results suggest that the local CVD of Haller's layer is unaffected in unilateral hyperopic amblyopic eyes.
\end{abstract}

Keywords: Amblyopia, Choroid, Vascular density, Optical coherence tomography

\footnotetext{
* Correspondence: amiki@tc5.so-net.ne.jp

'Department of Ophthalmology, Kawasaki Medical School, 577 Matsushima, Kurashiki, Okayama 701-0192, Japan

${ }^{3}$ Department of Sensory Science, Faculty of Health Science and Technology,

Kawasaki University of Medical Welfare, 288 Matsushima, Kurashiki, Okayama

701-0193, Japan

Full list of author information is available at the end of the article
}

(c) The Author(s). 2020 Open Access This article is licensed under a Creative Commons Attribution 4.0 International License, which permits use, sharing, adaptation, distribution and reproduction in any medium or format, as long as you give appropriate credit to the original author(s) and the source, provide a link to the Creative Commons licence, and indicate if changes were made. The images or other third party material in this article are included in the article's Creative Commons licence, unless indicated otherwise in a credit line to the material. If material is not included in the article's Creative Commons licence and your intended use is not permitted by statutory regulation or exceeds the permitted use, you will need to obtain permission directly from the copyright holder. To view a copy of this licence, visit http://creativecommons.org/licenses/by/4.0/. The Creative Commons Public Domain Dedication waiver (http://creativecommons.org/publicdomain/zero/1.0/) applies to the data made available in this article, unless otherwise stated in a credit line to the data. 


\section{Background}

Amblyopia is defined as a disorder with dysfunctional processing of visual information, such as reduced recognition visual acuity (VA), resulting from either disuse due to the absence of a clear image on the retina or misuse due to abnormal binocular interactions [1]. Traditionally, morphological abnormalities have not been considered present in the ocular structure of amblyopic eyes. In contrast, recent studies using optical coherence tomography (OCT) have shown structural changes to the choroid such as thickening in hyperanisometropic amblyopic eyes compared with that of fellow or healthy eyes, independent of axial length (AL) [2-5]. In chorioretinal diseases such as central serous chorioretinopathy [6] and Vogt-Koyanagi-Harada disease [7], the pathogenesis and clinical significance of choroidal structural changes is relatively well understood, and OCT findings are utilized for diagnosis and follow-up. In contrast, no consensus has been reached regarding the pathogenesis or clinical significance of choroidal structural changes in patients with amblyopia.

Recently, a new method other than thickness has been developed to evaluate choroidal structures, focusing on choroidal vasculature using binarization analyses [8, 9]. Some studies have suggested changes in choroidal vasculature in amblyopic eyes, but insufficient knowledge has been accumulated [10-13]. The purpose of the present study was thus to investigate the clinical significance of evaluating choroidal vascular density (CVD) in patients with amblyopia.

\section{Methods}

All investigative procedures adhered to the tenets of the Declaration of Helsinki. This study was approved by the Institutional Review Board Committee at Kawasaki Medical School (registration number: 3473). This study was designed as a retrospective observational case series and was conducted from November 2013 to December 2018 in the Department of Ophthalmology at Kawasaki Medical School Hospital. This study included minors under 16 years old among the patient sample. Informed consent for all examinations was obtained from one parent of each patient.

\section{Subjects}

Patients enrolled to this study showed unilateral amblyopia with decimal best-corrected visual acuities (BCVAs) $<0.8$ in the amblyopic eye and $>1.0$ in the fellow eye at the time of the first visit, due to hyperopic anisometropia with or without strabismus; and age $\leqq 18$ years. Hyperopic anisometropia was defined as hypermetropia in both eyes and an interocular difference in refraction (spherical equivalent) of $>1.5$ diopters (D). Treatment status was not considered at the time of performing swept-source OCT (SS-OCT) in this study. Exclusion criteria were as follows: presence of decimal BCVA in amblyopic eyes $>1.0$ within 2 months after full refractive correction or at the time of SS-OCT; history of ocular disease; history of intraocular surgery; or presence of systemic diseases that may have exerted an influence on the eye.

A total of 29 children were recruited as a normal control group. These children in the control group had a decimal BCVA $>1.0$ in both eyes, and no ocular or systemic diseases other than mild refractive errors. Only the right eye of the control group was used for data analysis.

Ophthalmologic examinations performed in all subjects included BCVA, cycloplegic refraction (measured using a RKT-7700 auto-refractor; NIDEK, Gamagori, Japan), AL (measured using an IOL Master OCT biometer; Carl Zeiss Meditec AG, Jena, Germany), cover test, extraocular movements, slit-lamp, fundoscopy, and SS-OCT (DRI OCT-1 Atlantis; Topcon Corporation, Tokyo, Japan). All SS-OCT examinations were performed by the same author (S.A.) between 9:00 AM and 12:00 PM under nonmydriatic conditions.

\section{Quantification of choroidal vascular density and subfoveal choroidal thickness}

CVD was quantified by binarizing the en-face image with reference to the report by Fujiwara et al. [9]. The 3dimensional (3D) SS-OCT images, which covered a $12 \times$ $9-\mathrm{mm}^{2}$ area with a scan density of $512 \times 256$, were reconstructed as en-face images flattened with Bruch's membrane using dedicated software tools (EnView version 1.0.1; Topcon Corporation). En-face images were extracted at the level where the distance from the inner surface of the choroid was $50 \%$ of the total subfoveal choroidal thickness (SFCT) to examine the CVD of Haller's layer (Fig. 1a).

The analyzed region of CVD was a $3 \times 3-\mathrm{mm}$ square centered on the fovea (Fig. 1b). The magnification error was corrected using the Littman and modified Bennett formulae, taking into account image magnification due to $\mathrm{AL}$ variation $[14,15]$. The extracted images were binarized by Niblack's method using ImageJ software (version 1.51; National Institutes of Health, Bethesda, $\mathrm{MD}$ ), and the percentage of black area (considered to represent the choroidal vascular area) in the total region of interest was calculated as CVD (Fig. 1c).

SFCT was defined as the vertical distance from the outer border of the hyperreflective line of retinal pigment epithelium/Bruch's complex to the choroidalscleral juncture at the subfovea. The SFCT was measured manually by S.A. from 3D SS-OCT images using the built-in caliper tool. Subjects with images that were 

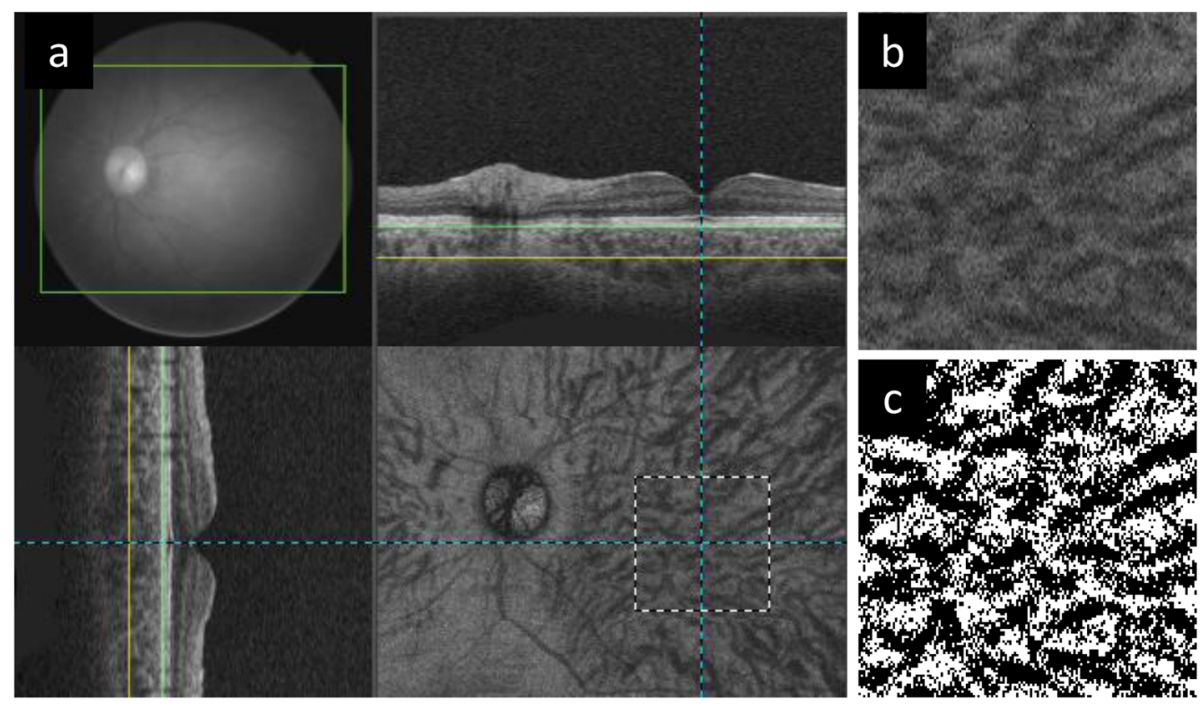

Fig. 1 Analysis of choroidal vascular density using binarized en-face images. a Three-dimensional swept-source optical coherence tomography images were reconstructed as en-face images flattened with Bruch's membrane. The yellow line shows where the subfoveal choroidal thickness is $50 \%$ level of total thickness. The white dotted-line shows the $3 \times 3-\mathrm{mm}$ square centered on the fovea after correction for magnification error using individual axial length. $\mathbf{b}$ Extracted $3 \times 3-\mathrm{mm}$ square en-face image. $\mathbf{c}$ En-face image after binarization. The black area is considered to be the choroidal vascular area, and the percentage within the region of interest is calculated as the choroidal vascular density

difficult to segment due to signal attenuation were excluded from the present study.

\section{Statistical analyses}

Data are presented as means \pm standard deviations. For statistical analysis, the decimal BCVA was converted to the logarithm of the minimal angle of resolution $(\log M A R)$.

First, multiple regression analysis of all eyes using the forced entry method was performed with CVD as a dependent variable, and $\log M A R$, refractive error (RE), and SFCT as independent variables to select those parameters to be corrected for comparisons of CVD. Because $\mathrm{AL}$ and $\mathrm{RE}$ are highly collinear and mutually confounding variables [16], only RE was used for the multiple regression analyses.

Second, a generalized linear mixed model (GLMM) was used for CVD comparisons with measured eye (amblyopic, fellow, or normal control eyes) as a fixed factor, individual subjects (amblyopic patients or healthy control children) and factors that were significant in multiple regression analysis as random factors. In addition, $\log$ MAR, RE, AL, and SFCT were compared among amblyopic, fellow and normal control eyes. If a significant difference was found by GLMM, pairwise comparison was performed with Bonferroni correction.

The two-sample t-tests and chi-squared test were used to compare age and sex ratio between patients with unilateral amblyopia and normal control groups, respectively. Pearson's correlation analysis was used to determine the coefficient of correlation between CVD and $\log$ MAR in amblyopic eyes.

A value of $p<0.05$ was considered statistically significant. All statistical analyses were conducted using SPSS Statistics version 20.0 (IBM Corporation, Somers, New York, USA).

\section{Results}

\section{Demographic data}

This study enrolled 44 patients with unilateral hyperopic amblyopia and 29 normal control individuals. All subjects were Japanese. Table 1 shows demographic and clinical data for all subjects. In the amblyopic group, mean age at the time of SS-OCT was $6.8 \pm 3.0 \mathrm{y}$ (range, 3-18 y), and 29 patients (66\%) were female. In the normal control group, mean age at the time of SS-OCT was $7.6 \pm 3.1 \mathrm{y}$ (range, $3-16 \mathrm{y}$ ), and 18 (62\%) individuals were female. No significant differences were evident between amblyopic and normal control groups with regard to age $(p=0.23)$ or sex ratio $(p=0.74)$.

Mean BCVA (logMAR) in amblyopic eyes was significantly worse than that of fellow or normal control eyes $(p<0.001$, each), with no significant difference between fellow and normal control eyes $(p=1.000)$. Mean RE in amblyopic eyes was more hyperopic than that of fellow and normal control eyes, and was more hyperopic in the fellow eyes than in the normal control eyes $(p<0.001$, $p<0.001$, and $p=0.003$, respectively). Mean AL in 
Table 1 Demographic and clinical data at SS-OCT measurements

\begin{tabular}{|c|c|c|c|c|c|c|}
\hline & $\mathrm{AE}(n=44)$ & FE $(n=44)$ & Cont. $(n=29)$ & $p$-value (AE vs FE) & $p$-value (AE vs Cont.) & $p$-value (FE vs Cont.) \\
\hline Age & $6.8 \pm 3.0$ & & $7.6 \pm 3.1$ & - & - & - \\
\hline Sex (Male: Female) & $15: 29$ & & 11: 18 & - & - & - \\
\hline Visual acuity (logMAR) & $0.38 \pm 0.22$ & $-0.13 \pm 0.07$ & $-0.12 \pm 0.07$ & $<0.001^{\mathrm{a}}$ & $<0.001^{\mathrm{a}}$ & $1.000^{\mathrm{a}}$ \\
\hline Refractive error (diopters) & $5.32 \pm 1.70$ & $1.68 \pm 1.31$ & $0.46 \pm 1.44$ & $<0.001^{\mathrm{a}}$ & $<0.001^{\mathrm{a}}$ & $0.003^{\mathrm{a}}$ \\
\hline Axial length (mm) & $21.00 \pm 0.98$ & $22.29 \pm 1.06$ & $22.77 \pm 1.11$ & $<0.001^{\mathrm{a}}$ & $<0.001^{\mathrm{a}}$ & $0.170^{\mathrm{a}}$ \\
\hline Subfoveal choroidal thickness ( $\mu \mathrm{m})$ & $387.6 \pm 78.4$ & $298.3 \pm 42.5$ & $323.8 \pm 69.1$ & $<0.001^{b}$ & $<0.001^{b}$ & $0.302^{b}$ \\
\hline
\end{tabular}

Values are shown as mean \pm standard deviation

$A E$ amblyopic eyes, FE fellow eyes, Cont. normal control eyes

${ }^{a}$ Bonferroni correction after GLMM

${ }^{b}$ Bonferroni correction after GLMM corrected with axial length

amblyopic eyes was significantly shorter than in fellow or normal control eyes $(p<0.001$, each), with no significant difference between fellow and normal control eyes $(p=0.170)$. Mean SFCT was significantly thicker in amblyopic eyes than in fellow or normal control eyes $(p<0.001$, each $)$, with no significant difference between fellow and normal control eyes $(p=0.302)$.

\section{CVD}

Mean CVD was $59.11 \pm 0.66 \%$ in amblyopic eyes, $59.23 \pm 0.81 \%$ in fellow eyes and $59.29 \pm 0.74 \%$ in normal control eyes (Fig. 2). Table 2 shows the results of multiple regression analysis of all eyes for CVD. SFCT was significantly associated with CVD (standardized $\beta=$ 0.307, $p=0.004)$. $\log$ MAR and RE were not associated factors with CVD. No significant difference in mean CVD was seen among amblyopic, fellow or normal control eyes with or without correction for SFCT $(p=0.502$, $p=0.505$, respectively; GLMM). In amblyopic eyes, no significant correlation existed between CVD and log$\operatorname{MAR}(r=0.094, p=0.545)$ (Fig. 3).

\section{Discussion}

The current findings showed that the CDV of Haller's layer, as analyzed by en-face OCT, did not differ significantly among amblyopic, fellow and normal control eyes. Also, no significant correlation was apparent between CVD and BCVA in amblyopic eyes.

The choroidal structure has been implicated in the pathogenesis of various ocular diseases. Agrawal et al. recently reported that choroidal vascularity index (CVI: the ratio of luminal area to total choroid area, equivalent to $\mathrm{CVD}$ ) is a robust marker for studying pathophysiologies of the choroid, because CVI is less variable than SFCT and is not associated with most physiological variables [17]. In fact, studies in patients with Stargardt

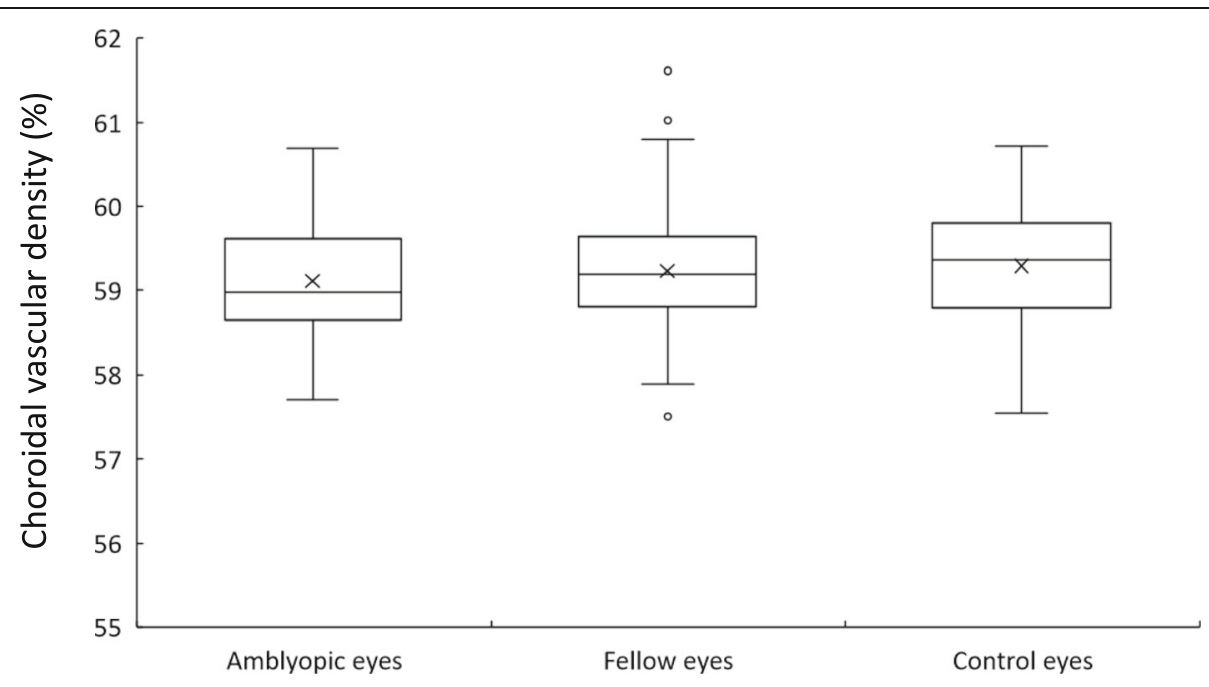

Fig. 2 Box plot of the choroidal vascular density in amblyopic, fellow and normal control eyes. Cross symbols show the mean values. Circle symbols show the outlier values which are more than 1.5 box lengths apart from either end of the box. There was no significant difference in the choroidal vascular density among the amblyopic, fellow and normal control eyes with or without correction for subfoveal choroidal thickness (amblyopic eyes vs. fellow eyes, $p=1.000, p=1.000$; amblyopic eyes vs. control eyes, $p=0.990, p=0.963$; fellow eyes vs. control eyes, $p=1.000$, $p=1.000$; Bonferroni correction) 
Table 2 Multiple regression analysis of all eyes for choroidal vascular density $(n=117)$

\begin{tabular}{llll}
\hline Independent variable & Standardized $\boldsymbol{\beta}$ & Partial correlation coefficient & $\boldsymbol{p}$-value \\
\hline LogMAR & -0.113 & -0.071 & 0.449 \\
Refractive error & -0.169 & -0.109 & 0.247 \\
Subfoveal choroidal thickness & 0.307 & 0.265 & 0.004 \\
\hline
\end{tabular}

disease [18] and retinopathy of prematurity [19] have found that CVI is a more sensitive biomarker than SFCT and that a decrease in CVI is associated with a decrease in BCVA. On the other hand, the present study observed no specific changes in the CVD of amblyopic eyes, and no correlation with BCVA, suggesting that CVD may not be an adequate biomarker for evaluating visual acuity loss in amblyopic patients. However, visual dysfunction in amblyopia can be evaluated using many parameters other than visual acuity. For example, amblyopic eyes show poorer accommodative performance than fellow or normal eyes [20]. Accommodation has been reported directly related to choroidal structure [21]. Therefore, novel findings may be obtained by using indices other than BCVA (e.g., accommodation) in the investigation of the relationship between amblyopia and CVD in the future.

Several reports have examined choroidal vascular structures in patients with amblyopia [10-13]. In reports using analyses by binarization of a single-section OCT image, Nishi et al. found that the luminal area was significantly larger and the stromal area was significantly smaller in amblyopic eyes than in control hyperopic eyes
[10]. Another study showed a reduction in the luminal area and widened stromal area after treatment only in amblyopic eyes [13]. Beak et al. reported that the ratio of choroidal luminal area to total choroidal area (i.e., CVD) of amblyopic eyes showed different characteristics from normal control eyes, such as amblyopic eyes showing higher CVD that did not increase in direct proportion to the increased choroidal thickness [12]. In contrast to those previous reports $[10,12,13]$, the present study did not find any difference in CVD between amblyopic patients and normal healthy subjects. Our study extracted en-face images at a fixed distance from Burch's membrane and analyzed the CVD. On the other hand, CVD analysis using B-scan images, as used in previous studies $[10,12,13]$, is able to analyze only one cross-section image, while allowing analysis of the structure of the inner and outer layers of choroid simultaneously. Differences in results between these studies may thus be due to differences in the methods used for CVD analysis.

On the basis of analyzing binarized en-face OCT images, Terada et al. reported that the outer choroidal vascular areas (i.e., CVD of Haller's layer) were larger in both amblyopic $(61.49 \pm 4.95 \%)$ and fellow eyes $(61.48 \pm$

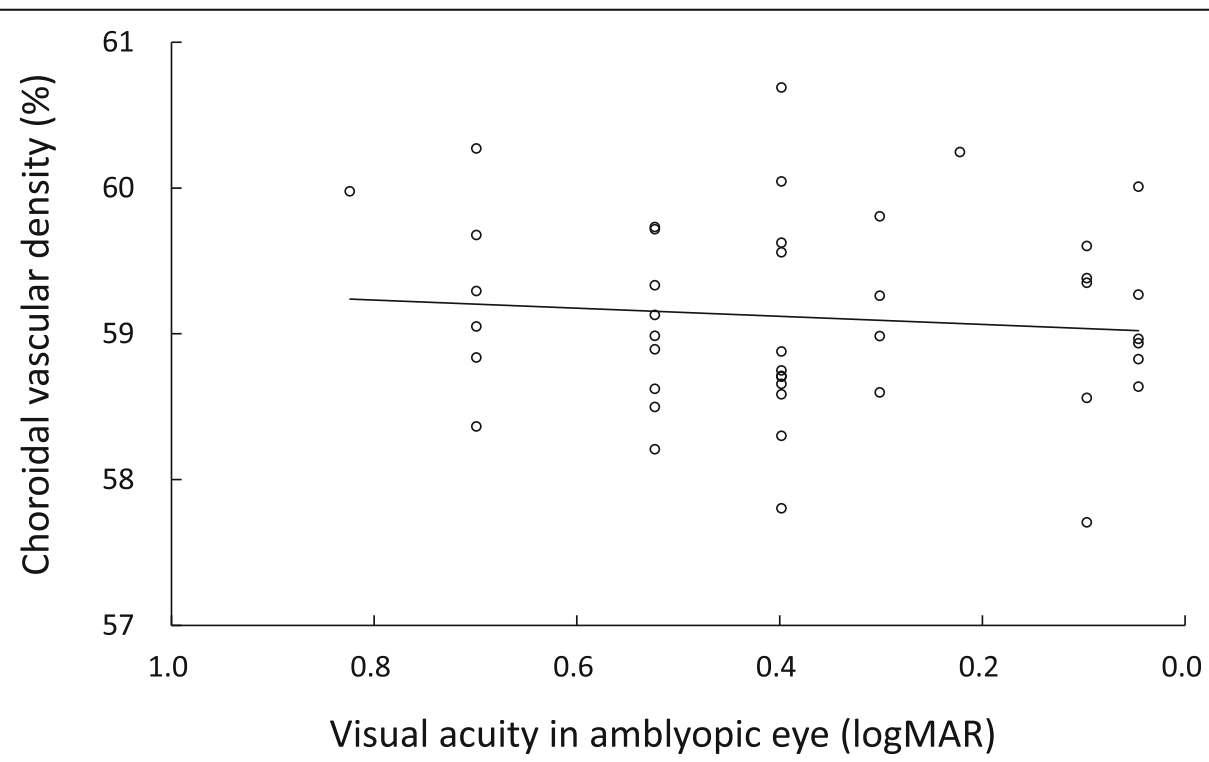

Fig. 3 Relationship between choroidal vascular density and best-corrected visual acuity in amblyopic eyes. No significant correlation is evident between choroidal vascular density and best-corrected visual acuity $(r=0.094, p=0.545)$ 
$3.73 \%)$ than in healthy eyes $(55.69 \pm 1.83 \%)$ [11]. They also indicated that the cutoff for distinguishing between amblyopic patients and controls was 59\%. In our current study, the CVD in amblyopic and fellow eyes exceeded $59 \%$ (59.11 $\pm 0.66 \%$ and $59.23 \pm 0.81 \%$, respectively), consistent with a report by Terada et al. However, the CVD in normal control eyes $(59.29 \pm 0.74 \%)$ also exceeded $59 \%$, resulting in comparable CVDs among different eye conditions. In normal eyes, CVD has been reported to correlate positively with SFCT [9]. However, SFCT was slightly thicker in Terada's control group (351.9 \pm $60.7 \mu \mathrm{m})$ than in our control group $(323.8 \pm 69.1 \mu \mathrm{m})$. The higher CVD in the normal control eye is thus not attributable to differences in SFCT. Substantial individual differences might be present in CVD. We should therefore be cautious of using CVD analysis of en-face OCT images to evaluate amblyopia.

The present study showed that SFCT was significantly thicker in the amblyopic eye than in fellow and normal control eyes, while CVD was comparable among all eyes. The results indicate that choroidal blood flow with respect to total choroidal volume may be increased in amblyopic eyes than in fellow and normal control eyes. A possible future direction of this work would be to investigate the ratio of choroidal vessels to total volume of the choroid in amblyopic eyes.

Several limitations should be considered when interpreting the findings of the present study. First, the sample size was small. A further study of a larger number of patients with detailed classifications of the degree and type of amblyopia will need to be undertaken to validate our findings. Second, CVD could only be assessed in a $3 \times 3-\mathrm{mm}$ localized area centered on the fovea. This was due to the large difference in thickness between the center and periphery of the choroid, making it difficult to capture the same depth of vascular structure in the center and periphery if the analyzed area of the en-face image flattened with Bruch's membrane is expanded. In the future, new tools, such as high-penetration doppler optical coherence angiography [22] and hybrid threedimensional models of the choroidal vasculature [23], are expected to provide a more detailed assessment of choroidal vascular structures.

\section{Conclusions}

In conclusion, the CVD of Haller's layer did not differ significantly between amblyopic eyes and fellow or normal control eyes. Evaluation of CVD using en-face OCT may have little clinical significance in elucidating the mechanisms underlying amblyopia.

\section{Abbreviations}

AL: Axial length; BCVA: Best-corrected visual acuity; CVD: Choroidal vascular density; CVl: Choroidal vascularity index; GLMM: Generalized linear mixed model; logMAR: Logarithm of the minimal angle of resolution;
SFCT: Subfoveal choroidal thickness; SS-OCT: Swept-source optical coherence tomography; RE: Refractive error

\section{Acknowledgements}

Not applicable.

\section{Authors' contributions}

SA and AM designed the study, collected and interpreted the data, drafted the manuscript, and reviewed the literature. KG, TY1, TY2, and KH participated in the collection and interpretation of the data. SA and KY performed statistical analysis. AF, YI, JK, and GM interpreted the data, and critically reviewed the manuscript. All authors read and approved the final manuscript.

\section{Funding}

The Japan Society for the Promotion of Science (JPSP) provided financial support in the form of KAKENHI Grants-in-Aid (Grant Number: 17 K04506, Grant Recipient: Goro Maehara). The funding provided support for analysis of the data.

\section{Availability of data and materials}

The datasets used and/or analysed during the current study are available from the corresponding author on reasonable request.

\section{Ethics approval and consent to participate}

This study adhered to the tenets of the Declaration of Helsinki and was approved by the Institutional Review Board committee of Kawasaki Medical School (registration number: 3473). Additionally, oral informed consent for the examinations was obtained from each patient. If the patient was a minor (under the age of 16), informed consent for the examinations was obtained from one of the parents. The institutional review board committee at Kawasaki Medical School did not require written informed consent for this study as it was a non-invasive observational studies using only existing clinical data.

\section{Consent for publication}

Not applicable.

\section{Competing interests}

The authors declare that they have no competing interests.

\section{Author details}

'Department of Ophthalmology, Kawasaki Medical School, 577 Matsushima, Kurashiki, Okayama 701-0192, Japan. ²Doctoral Program in Sensory Science, Graduate School of Health Science and Technology, Kawasaki University of Medical Welfare, 288 Matsushima, Kurashiki, Okayama 701-0193, Japan.

${ }^{3}$ Department of Sensory Science, Faculty of Health Science and Technology, Kawasaki University of Medical Welfare, 288 Matsushima, Kurashiki, Okayama 701-0193, Japan. ${ }^{4}$ Division of Ophthalmology and Visual Sciences, Niigata University Graduate School of Medical and Dental Sciences, 1-757 Asahimachi-dori, Chuo-ku, Niigata, Niigata 951-8510, Japan. ${ }^{5}$ Field of Orthoptics and Visual Sciences, Major in Medical and Rehabilitation Sciences, Graduate School of Health and Welfare, Niigata University of Health and Welfare, 1398 Shimami-cho, Kita-ku, Niigata, Niigata 950-3198, Japan. ${ }^{6}$ Department of Human Sciences, Kanagawa University, 3-27-1 Rokkakubashi, Yokohama, Kanagawa 221-8686, Japan. ${ }^{7}$ Yaoeda Eye Clinic, 2-1649-1 Naga-Chou, Nagaoka, Niigata 940-0053, Japan.

Received: 4 August 2020 Accepted: 17 November 2020

Published online: 02 December 2020

\section{References}

1. Holmes JM, Clarke MP. Amblyopia. Lancet. 2006;367(9519):1343-51.

2. Nishi T, Ueda T, Hasegawa T, Miyata K, Ogata N. Choroidal thickness in children with hyperopic anisometropic amblyopia. Br J Ophthalmol. 2014;98: 228-32.

3. Liu Y, Dong Y, Zhao K. A meta-analysis of Choroidal thickness changes in unilateral amblyopia. J Ophthalmol. 2017;2017:2915261.

4. Araki S, Miki A, Goto K, Yamashita T, Takizawa G, Haruishi K, et al. Macular retinal and choroidal thickness in unilateral amblyopia using swept-source optical coherence tomography. BMC Ophthalmol. 2017;17(1):167. 
5. Araki S, Miki A, Goto K, Yamashita T, Takizawa G, Haruishi K, et al. Effect of amblyopia treatment on choroidal thickness in hypermetropic anisometropic amblyopia using swept-source optical coherence tomography. BMC Ophthalmol. 2018;18(1):227.

6. Imamura Y, Fujiwara T, Margolis R, Spaide RF. Enhanced depth imaging optical coherence tomography of the choroid in central serous chorioretinopathy. Retina. 2009;29(10):1469-73.

7. Maruko I, lida T, Sugano Y, Oyamada H, Sekiryu T, Fujiwara T, et al. Subfoveal choroidal thickness after treatment of Vogt-Koyanagi-Harada disease. Retina. 2011;31(3):510-7.

8. Sonoda S, Sakamoto T, Yamashita T, Shirasawa M, Uchino E, Terasaki H, et al. Choroidal structure in normal eyes and after photodynamic therapy determined by binarization of optical coherence tomographic images. Invest Ophthalmol Vis Sci. 2014;55(6):3893-9.

9. Fujiwara A, Morizane Y, Hosokawa M, Kimura S, Kumase F, Shiode Y, et al. Factors affecting Choroidal vascular density in Normal eyes: quantification using en face swept-source optical coherence tomography. Am J Ophthalmol. 2016;170:1-9.

10. Nishi T, Ueda T, Mizusawa Y, Shinomiya K, Semba K, Mitamura Y, et al. Choroidal structure in children with Anisohypermetropic amblyopia determined by Binarization of optical coherence tomographic images. PLoS One. 2016:11(10):e0164672.

11. Terada N, Miyata M, Muraoka Y, Hata M, Fujimoto M, Yokota S, et al. Abnormal outer Choroidal vasculature in amblyopia. J Ophthalmol. 2019; 2019:2097087.

12. Baek J, Lee A, Chu M, Kang NY. Analysis of Choroidal vascularity in children with unilateral hyperopic amblyopia. Sci Rep. 2019;9(1):12143.

13. Nishi T, Ueda T, Mizusawa Y, Semba K, Shinomiya K, Mitamura Y, et al. Effect of optical correction on choroidal structure in children with anisohypermetropic amblyopia. PLoS One. 2020;15(4):e0231903.

14. Bennett AG, Rudnicka AR, Edgar DF. Improvements on Littmann's method of determining the size of retinal features by fundus photography. Graefes Arch Clin Exp Ophthalmol. 1994;232(6):361-7.

15. Sampson DM, Gong P, An D, Menghini M, Hansen A, Mackey DA, et al. Axial length variation impacts on superficial retinal vessel density and Foveal avascular zone area measurements using optical coherence tomography angiography. Invest Ophthalmol Vis Sci. 2017;58(7):3065-72.

16. Nishida Y, Fujiwara T, Imamura Y, Lima LH, Kurosaka D, Spaide RF. Choroidal thickness and visual acuity in highly myopic eyes. Retina. 2012;32(7):1229-36.

17. Agrawal R, Gupta P, Tan KA, Cheung CM, Wong TY, Cheng CY. Choroidal vascularity index as a measure of vascular status of the choroid: measurements in healthy eyes from a population-based study. Sci Rep. 2016;6:21090.

18. Ratra D, Tan R, Jaishankar D, Khandelwal N, Gupta A, Chhablani J, et al. CHOROIDAL structural changes and vascularity index in STARGARDT disease on swept source optical coherence tomography. Retina. 2018:38(12):2395-400.

19. Lavric A, Tekavcic Pompe M, Markelj S, Ding J, Mahajan S, Khandelwal N, et al. Choroidal structural changes in preterm children with and without retinopathy of prematurity. Acta Ophthalmol. 2019. https://doi.org/10.1111/ aos.14324.

20. Manh V, Chen AM, Tarczy-Hornoch K, Cotter SA, Candy TR. Accommodative performance of children with unilateral amblyopia. Invest Ophthalmol Vis Sci. 2015;56(2):1193-207.

21. Woodman-Pieterse EC, Read SA, Collins MJ, Alonso-Caneiro D. Regional changes in Choroidal thickness associated with accommodation. Invest Ophthalmol Vis Sci. 2015;56(11):6414-22.

22. Hong YJ, Miura M, Makita S, Ju MJ, Lee BH, Iwasaki T, et al. Noninvasive investigation of deep vascular pathologies of exudative macular diseases by high-penetration optical coherence angiography. Invest Ophthalmol Vis Sci. 2013;54(5):3621-31.

23. Sekiryu T, Sugano Y, Ojima A, Mori T, Furuta M, Okamoto M, et al. Hybrid three-dimensional visualization of Choroidal vasculature imaged by sweptsource optical coherence tomography. Transl Vis Sci Technol. 2019;8(5):31.

\section{Publisher's Note}

Springer Nature remains neutral with regard to jurisdictional claims in published maps and institutional affiliations.

\section{Ready to submit your research? Choose BMC and benefit from:}

- fast, convenient online submission

- thorough peer review by experienced researchers in your field

- rapid publication on acceptance

- support for research data, including large and complex data types

- gold Open Access which fosters wider collaboration and increased citations

- maximum visibility for your research: over $100 \mathrm{M}$ website views per year

At BMC, research is always in progress.

Learn more biomedcentral.com/submissions 